\title{
GAIA Level 3b Failure to Thrive
}

National Cancer Institute

\section{Source}

National Cancer Institute. GAIA Level 3b Failure to Thrive. NCI Thesaurus. Code C128774.

GAIA Level 3b Failure to Thrive is defined by three criteria: first, infants have no weight available; second, physical examination is consistent with failure to thrive, which is defined as having at least two findings, one of which must be a major finding: reduced subcutaneous fat stores, poor muscle mass, loose skin folds, prominent ribs, and thin limbs. Less specific findings include sparse hair, rashes, pallor, irritable temperament, and lethargy/fatigue; third, a mid-upper arm circumference (MUAC) measurement, which is indicative of severe wasting, that is less than or equal to $110 \mathrm{~mm}$ in infants up to six months of age, and that is less than or equal to $115 \mathrm{~mm}$ in infants six to twelve months of age. 